\title{
Adaptive Imaging Using the Generalized Coherence Factor
}

\author{
Pai-Chi Li, Senior Member, IEEE, and Meng-Lin Li, Student Member, IEEE
}

\begin{abstract}
Sound-velocity inhomogeneities degrade both spatial and contrast resolutions. This paper proposes a new adaptive imaging technique that uses the generalized coherence factor (GCF) to reduce the focusing errors resulting from the sound-velocity inhomogeneities. The GCF is derived from the spatial spectrum of the received aperture data after proper receive delays have been applied. It is defined as the ratio of the spectral energy within a prespecified low-frequency range to the total energy. It is demonstrated that the low-frequency component of the spectrum corresponds to the coherent portion of the received data and that the high-frequency component corresponds to the incoherent portion. Hence, the GCF reduces to the coherence factor defined in the literature if the prespecified lowfrequency range is restricted to dc only. In addition, the GCF is also an index of the focusing quality and can be used as a weighting factor for the reconstructed image. The efficacy of the GCF technique is demonstrated for focusing errors resulting from the sound-velocity inhomogeneities. Simulations and real ultrasound data are used to evaluate the efficacy of the proposed GCF technique. The characteristics of the GCF, including the effects of the signal-to-noise ratio and the number of channels, are also discussed. The GCF technique also is compared with the correlation-based technique and the parallel adaptive receive compensation algorithm; the improvement in image quality obtained with the proposed technique rivals that of the latter technique. In the presence of a displaced phase screen, this proposed technique also outperforms the correlation-based technique. Computational complexity and implementation issues also are addressed.
\end{abstract}

\section{INTRODUCTION}

$\mathrm{R}$ EMOVING focusing errors resulting from soundvelocity inhomogeneities has been an important research topic [1]-[6]. These inhomogeneities distort both the amplitude and phase of the acoustic signal. Such soundvelocity inhomogeneities also are known as phase aberrations, and many methods have been proposed to restore the degraded resolution. Flax and O'Donnell [1] proposed modeling the sound-velocity inhomogeneities as a nearfield phase screen. Under this assumption, the inhomogeneities simply produce time-delay errors that can be estimated by cross-correlating the signals from adjacent channels [1], [2], or those from individual channels and the beam sum [3]. A similar approach models sound-velocity inhomogeneities as a phase screen displaced from the transducer surface [4]. Acoustic signals then are backpropagated to an

Manuscript received July 16, 2002; accepted October 3, 2002. This work was partially supported by Siemens.

The authors are with the Department of Electrical Engineering, National Taiwan University. optimal depth before the correlation function is calculated. The near-field, phase-screen model also can be generalized to distributed sound-velocity inhomogencities. In this case, the received signal is decomposed into spectral subbands before the time delays are estimated [5]. Although the specific details are different, all the above methods use the correlation function to find time-delay errors. Unfortunately, the usual requirement for a two-dimensional array to avoid integration errors has limited the widespread application of such approaches, especially in clinical applications [6].

Focusing imperfections reduce the cohcrence of the received signal and elevate the sidelobes of the radiation pattern. Therefore, the development of an adaptive sidelobereduction scheme is desirable. One such example is the parallel adaptive receive compensation algorithm (PARCA) technique [7]-[10]. This technique does not make assumptions regarding the source of the focusing errors. Instead, it directly estimates and reduces the unwanted sidelobe contribution (i.e., the contribution of off-axis scatterers to the on-axis signal). The PARCA approach requires the use of parallel receive beams around a fixed transmit beam (also known as single transmit imaging [11]) to estimate the sidelobe contribution. To estimate the sidelobe contribution, the original version of PARCA also requires the application of the total least-squares (TLS) method for every point in the image [7]; hence, it is computationally demanding. To simplify the technique, a modified version called PARCA2 was proposed [10]. Here the formation of parallel beams is approximated by Fourier transforming the aperture data (i.e., the received channel data along the array direction), and an iterative scheme is used to replace the TLS method [10], [12]. In addition, based on the relationship between parallel receive beams and the Fourier spectrum over the aperture, the sidelobe filter and the null filter were proposed to improve the beam quality with a point target [13]. These filters suppress the sidelobe level by scaling the received signal according to the ratio of the amplitude of the received signal along the transmit beam direction to the total sidelobe amplitude. The filter properties are determined by two adjustable parameters: the filter order and a scaling factor. The scaling ratio generally can be viewed as the square root of the coherence factor [14].

This paper proposes a more efficient approach for adaptive imaging based on the spatial spectrum of the aperture data. Specifically, the spectrum of the aperture data (i.e., the channel data along the array direction) is used to determine the received signal coherence relative to the incoherence. The signal cohcrence was described in the literature 
and is also known as the coherence factor (CF) [14]. It is defined as:

$$
\mathrm{CF}=\frac{\left|\sum_{i=0}^{N-1} S(i)\right|^{2}}{N \sum_{i=0}^{N-1}|S(i)|^{2}},
$$

where $S(i)$ is the received signal of channel $i$ after proper receive delays have been applied, and $N$ is the total number of array channels [14]-[16]. Note that the time index is omitted in (1). The numerator in (1) represents the energy of the coherent sum obtained in a conventional delayand-sum based beamformer, and the denominator is interpreted as the total incoherent energy that is $N$ times the incoherence sum. The CF can be used as an index of focusing quality. In this paper, such a notion is adopted and applied to improve the beam quality. Specifically, the coherence factor will be generalized to cover objects with diffuse scatterers. Moreover, the generalized coherence factor (GCF) will be used as an adaptive weighting factor to each image point. Simulations and real ultrasound data will be used to evaluate the efficacy of the proposed GCF technique for both point targets and diffuse scatterers. Contrast resolution and other general characteristics of the GCF technique also will be explored.

The paper is organized as follows. Frequency-domain interpretation of the apcrture data is described in Section II. The GCF-based adaptive weighting approach is introduced in Scction III. Performance of the proposed technique for compensation of sound-velocity inhomogeneities is evaluated in Section IV, and the implementation issues are discussed in Section V. Characteristics of the GCF also are explored and compared to those of the original CF. Moreover, the effectivencss of the proposed technique in the presence of a displaced phase screen is compared with existing approaches. The paper concludes in Section VI.

\section{FrequenCy-DOMAin INTERPRETATION OF THE APERTURE DATA}

The channcl data is the data received by each array channel after the focusing delays are applied prior to the beam sum. At a particular range, the time index is fixed and, hence, can be omitted. At this range, the data received by each channel $i$ across the array also is called the aperture data, and can be denoted $S(i)$ for $i=0$ to $N-1$, where $N$ is the total number of array channels. The $N$-point discrete Fourier spectrum of the aperture data becomes:

$$
\begin{aligned}
p(k) & =\sum_{i=0}^{N-1} S(i) e^{-j 2 \pi\left(i-\frac{N}{2}\right) d \frac{k}{N d}} \\
& =e^{j \pi k} \sum_{i=0}^{N-1} S(i) e^{-j 2 \pi \frac{i k}{N}},
\end{aligned}
$$

where $k=0$ to $N-1$ is the spatial frequency index, and $d$ is the pitch of the array with the array center defined as the zero-phase reference point. As described in [10], the Fourier spectrum across the array can be viewed as the approximation of multiple parallel receive beams centered at the transmit beam direction and equally spaced by $\Delta \sin \theta=\lambda /(N d)$, where $\theta$ is the steering angle in a sector scan and $\lambda$ is the wavelength. The direct current (DC) component (i.e., $p(0)=\sum_{i=0}^{N-1} S(i)$ ) representing the coherent sum after typical delay-and-sum operations corresponds to the received signal from the transmit beam direction, and the high-frequency components correspond to the scattered signals from other angles than the transmit beam direction.

The top panels of Fig. 1 show the channel data and the associated spectra across the receive array for a focused point target located at $60 \mathrm{~mm}$. In Fig. 1 and the subsequent simulations, the phased array had 64 elements, with a center frequency of $3.5 \mathrm{MH} z$ and a half-wavelength pitch. The top-left panel in Fig. 1 shows the channel data, and the top-middle pancl shows the corresponding onedimensional Fourier transform of the aperture data at each range, with the horizontal axis representing either the spatial frequency index $k$ from $-N / 2$ to $N / 2-1$ or $\sin (\theta)$ from -1 to $1-2 / N$ (from left to right). Note that the channel data is demodulated to baseband and filtered before the Fourier transform is calculated along the array direction. Because all of the array channels receive identical data from a focused point target, the aperture data at a given range are constant. Hence, the spectrum of such aperture data becomes a sinc function. The sinc function also is identical to the continuous-wave radiation pattern of the same aperture, except that the index $k$ is now related to the stcering angle rather than the spatial frequency. The top-right panel is the projected spectrum of the middle panel along the range direction, with each vertical division representing $10 \mathrm{~dB}$ and the middle vertical line indicating DC (i.e., the transmit beam direction). Note that the maximum along the range direction is used for the projection.

The second row of Fig. 1 demonstrates the case in which the point is moved to $9^{\circ}$ from the center line with the other conditions being unchanged. This corresponds to a stecring error of the receive array. It can be seen that the received channel data are no longer in phase, with the slope of the channel data corresponding to the direction of the point target. Thus, the aperture data at a particular range is modulated, and the corresponding spectrum across the array becomes a shifted sinc function, as shown in the middle and the right panels. It is clearly shown that, when a steering error is present, the energy in the coherent sum (i.e., the DC component) is non-negligible, although this energy is primarily concentrated in the object direction. In other words, the DC component (i.e., the beam sum) is the sidelobe contribution from the off-angle target, and this is what needs to be reduced as much as possible.

The bottom panels of Fig. 1 show the results in which the focal point is moved to $30 \mathrm{~mm}$, and the point target is still located at $60 \mathrm{~mm}$ (i.e., a range focusing error). There is again a significant portion of the received signal in the 


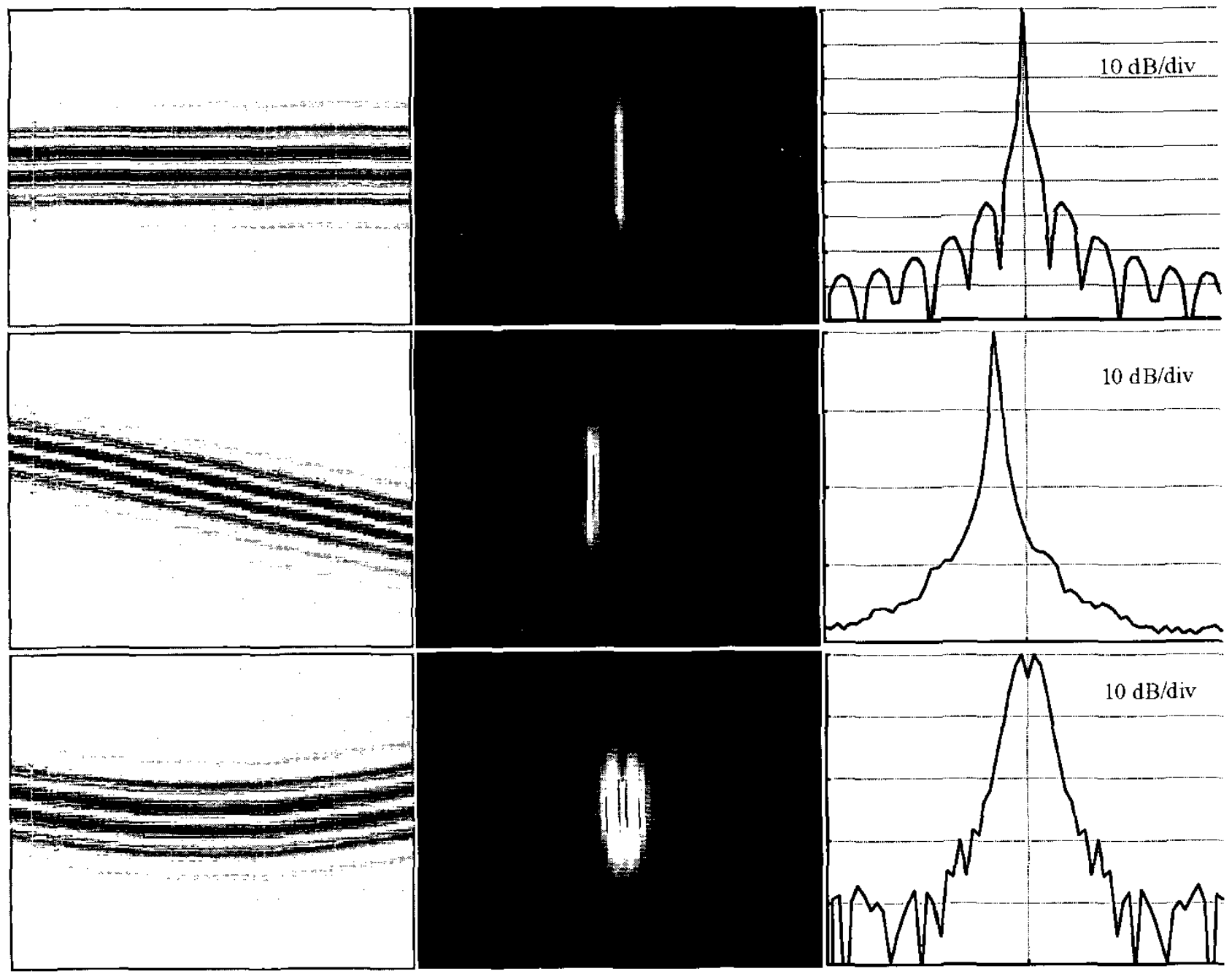

Fig. 1. Channel data and the corresponding spectrum over the aperture of a point target. The top panels are with ideal focusing, the middle panels are with a steering error, and the bottom panels are with a range focusing error. In each case, the left panel shows the channel data, with the horizontal axis representing the channel index and the vertical axis denoting the range. The middle panel shows the spectrum at each range. The right panel is the projection of the data shown in the middle panel.

non-DC portion of the spectrum (i.e., the angles outside of the transmit beam direction). Fig. 1 demonstrates the spectrum of the aperture data can be used to determine the focusing quality. The focusing quality is directly related to the ratio of the energy of the coherent sum to the total energy (i.e., the ratio of the energy of the received signal from the transmit beam direction to the total energy from all directions). Because the total spectral energy is $N$ times the incoherent sum according to the Parseval's relation for the discrete Fourier transform, which can be expressed as:

$$
\sum_{k=0}^{N-1}|p(k)|^{2}=N \cdot \sum_{i=0}^{N-1}|S(i)|^{2}
$$

where $S(i)$ is the signal received by channel $i[17]$, the ratio is the CF defined in (1). Intuitively, if the focusing quality is good, the energy from the transmit beam direction is high (i.e., the energy is more concentrated at the DC).
That is, good focusing quality results in a higher CF. In addition, the DC portion is also the coherent sum and the non-DC portion is rclated to the incoherent sum. Hence, the CF (or similar quantities) can be used as an index of the focusing quality and the basis of the proposed adaptive weighting technique. Also note that the coherent and incoherent sums are related to the main- and sidelobes of the radiation pattern. Thus, the CF also represents the ratio of mainlobe energy to total energy.

Focusing errors due to sound-velocity inhomogeneities also are considered, using the normalized phase-aberration profile shown in Fig. 2. The horizontal axis is the channel index and the vertical axis shows the phase error after normalization. The profile has a correlation length of $5 \mathrm{mum}$, and the maximum phase error varies depending on specific simulation conditions. The results are shown in Fig. 3. The top panels of Fig. 3 show a maximum phase error of $\pi / 4$; the middle panels show a maximum phase error of $\pi / 2$. 


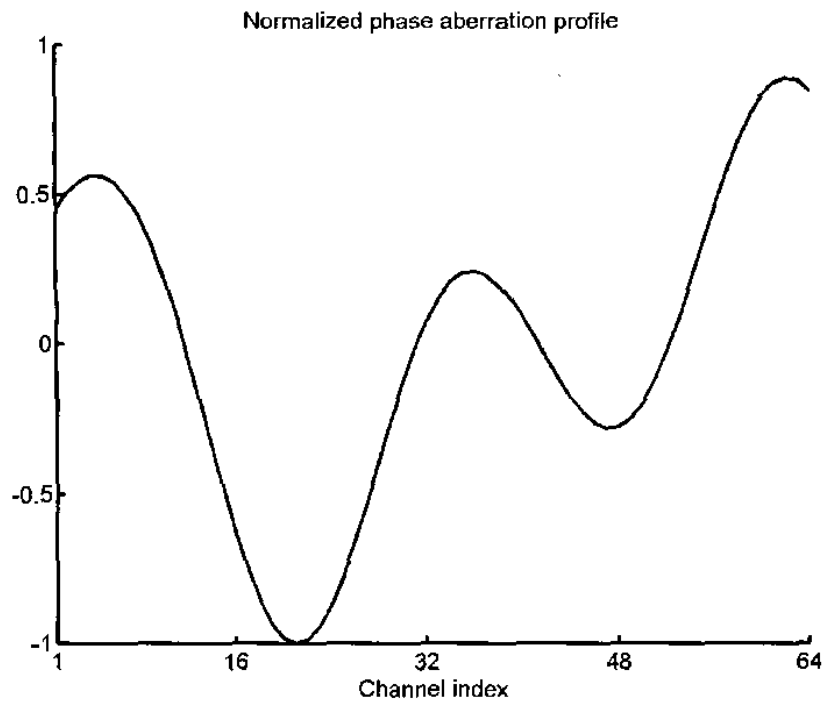

Fig. 2. The normalized phase-aberration profile used in the simulations of sound-velocity inhomogeneities. The horizontal axis is the channel index, and the vertical axis is the normalized phase error. 'The correlation length is $5 \mathrm{~mm}$.

Both conditions are for the near-field assumption, i.e., the time-delay errors occur at the face of the transducer. In the bottom panels, the same aberration profile used in the middle panels (i.e., $\pi / 2$ maximum phase error) is moved to $20 \mathrm{~mm}$ away from the transducer. Fig. 3 shows that the $\mathrm{CF}$ decreases as the maximum aberration increases. However, the coherence for the case with a displaced phase screen is not necessarily lower than that for the near-field case. In other words, although the displaced phase screen generally decreases the similarity between signals received by adjacent channels, this does not necessarily result in a reduction in the signal coherence as determined by the spectrum of the aperture data.

The results shown in Fig. 3 are for point targets; in clinical situations, speckle-gcnerating targets are more likely to occur. Fig. 4 shows the results with a speckle-generating target, in which the top panels are for perfect focusing, and the middle and bottom panels are with the same aberration conditions as the ones used in the middle and bottom panels of Fig. 3, respectively. Although the general trend is still the same as in Fig. 3, in Fig. 4 the mainlobe generally becomes wider and the non-DC level also increases for a speckle-generating target. This is because a spccklegenerating target inherently contains a certain degree of incoherence. Thus, with diffuse scatterers, the definition of the CF needs to be modified to take such inherent incoherence of speckle-generating targets into consideration.

\section{Adaptive Weighting Based on the GCF}

Section II demonstrates that the effects of focusing errors are directly related to the ratio of the energy of the coherent portion of the received data to the total energy, and so an adaptive weighting technique can be developed based on this property. When the ratio is high, the focusing quality is good; thus the weighting is high so that image intensity is maintained. However, the focusing quality is low when the ratio is low; hence, the weighting needs to be low to reduce artifacts. Furthermore, because the speckle-generating target inherently contains a certain degree of incoherence, the notion of CF is now generalized and will be referred to as the GCF. The GCF is derived from the spectrum of the aperture data after proper receive delays have been applied. Specifically, the GCF is defined as the ratio of the spectral energy within a prespecified low-frequency region to the total energy (i.e., the ratio of the energy received from the angles around the transmit beam direction to the total energy from all directions). The GCF over the aperture at a given range can be expressed as:

$$
\begin{aligned}
\mathrm{GCF} & =\frac{\text { energy in a low-frequency region }}{\text { total energy }} \\
& =\frac{\sum_{k \in \text { low-frcquency region }}|p(k)|^{2}}{\sum_{k=0}^{N-1}|p(k)|^{2}}
\end{aligned}
$$

where $p(k)$ is the spectrum of the received array data, and $N$ is the number of points in the discrete spectrum. The discrete Fourier transform can be efficiently computed using the fast Fourier transform (FFT), and $N$ is equal to the number of channels in this case. The low-frequency region is specified by a cutoff frequency $M_{0}$ in the spatial frequency index (i.e., from $-M_{0}$ to $M_{0}$ ). The low-frequency region can be viewed as the received signal from the angles around the transmit beam direction. Note that an $M_{0}$ of zero means that the low-frequency range used to estimate GCF is restricted to DC only. The procedure is illustrated in Fig. 5(a).

Note that, although derivation of the GCF requires the individual aperture data, the adaptive weighting only needs to be applied at each image point. In other words, the corrected signal $x_{\text {corrected }}$ of $x$ at a given range can be expressed as:

$$
x_{\text {corrected }}=\mathrm{GCF} \cdot x \text {. }
$$

A system block diagram for the proposed GCF technique is shown in Fig, 5(b). After the echo signal is received and digitized by the A/D converter, the received RF data are demodulated down to baseband. The baseband beamformer applies proper dynamic receive delays and phase rotations to the baseband data before the data are stored in the channel buffer. The GCF is then estimated using the delayed baseband array data across the aperture at each range, and the GCF should be calculated for all range points. Then, the beam sum data are weighted by GCF. The weighting is done by multiplying the amplitude of the beam sum data by the corresponding GCF. The weighted data then are sent to the beam buffer for further signal processing, scan conversion, and display. Note that GCF can be efficiently computed via the FFT. The computational 


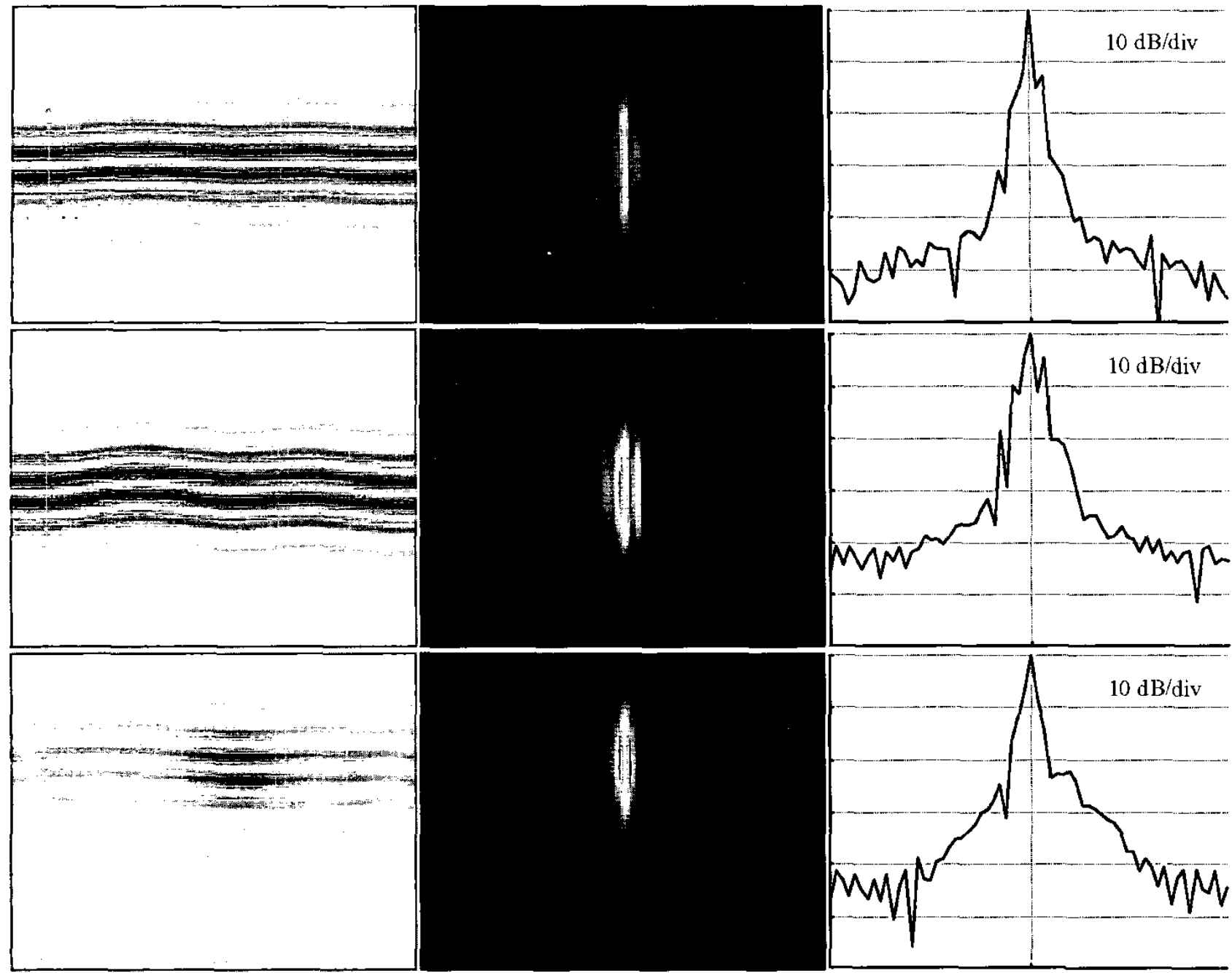

Fig. 3. Channel data and the corresponding spectrum over the aperture of a point target. The top panels are for a maximum phase crror cof $\pi / 4$, and the middle and bottom panels are for a maximum phase error of $\pi / 2$. The top and middle paneis are for a near-field phase screen; the bottom panels are for a displaced phase screen that is positioned $20 \mathrm{~mm}$ from the transducer. In each case, the left panel shows the channel data, with the horizontal axis representing the charmel index and the vertical axis denoting the range. The middle panel shows the spectrum at each range. The right panel is the projection of the data shown in the middle panel.

complexity is relatively low, and no iteration is needed. In addition, as illustrated in Fig. 5(b), the proposed technique easily can be incorporated into a baseband beamformer.

\section{Simulation and Experimental Results}

In this section, simulated ultrasound data are uscd to explore the efficacy of the GCF-weighting technique in compensating for the focusing errors resulting from sound-velocity inhomogeneities. The acoustic field simulation program Field II was used to simulate the received signal at each array element [18]. The simulated phased array had 64 elements with a center frequency of $3.5 \mathrm{MHz}$ and a half-wavelength pitch. A sector scan from $-30^{\circ}$ to $30^{\circ}$ was performed and Nyquist beam spacing was used. Two types of image objects - a point target and an anechoic object- were simulated. In addition to simulated data, emulated images using real ultrasound data also are presented.

\section{A. Point Target Simulation}

A point target simulation with focusing errors due to sound-velocity inhomogeneities is used to evaluate the GCF technique and effects of the parameter $M_{0}$. Only a near-field phase screen was simulated for all aberrated cases. The used normalized aberration profile is the same as that shown in Fig. 2. For all aberrated cases, the aberration profile is applied on both transmit and receive. The transmit focal point is $30 \mathrm{~mm}$ from the transducer, and dynamic focusing is applied on receive. The point target is located at the depth of $30 \mathrm{~mm}$. Fig. 6 shows the projected beam patterns for the point target under different aberration conditions and different values of $M_{0}$. 


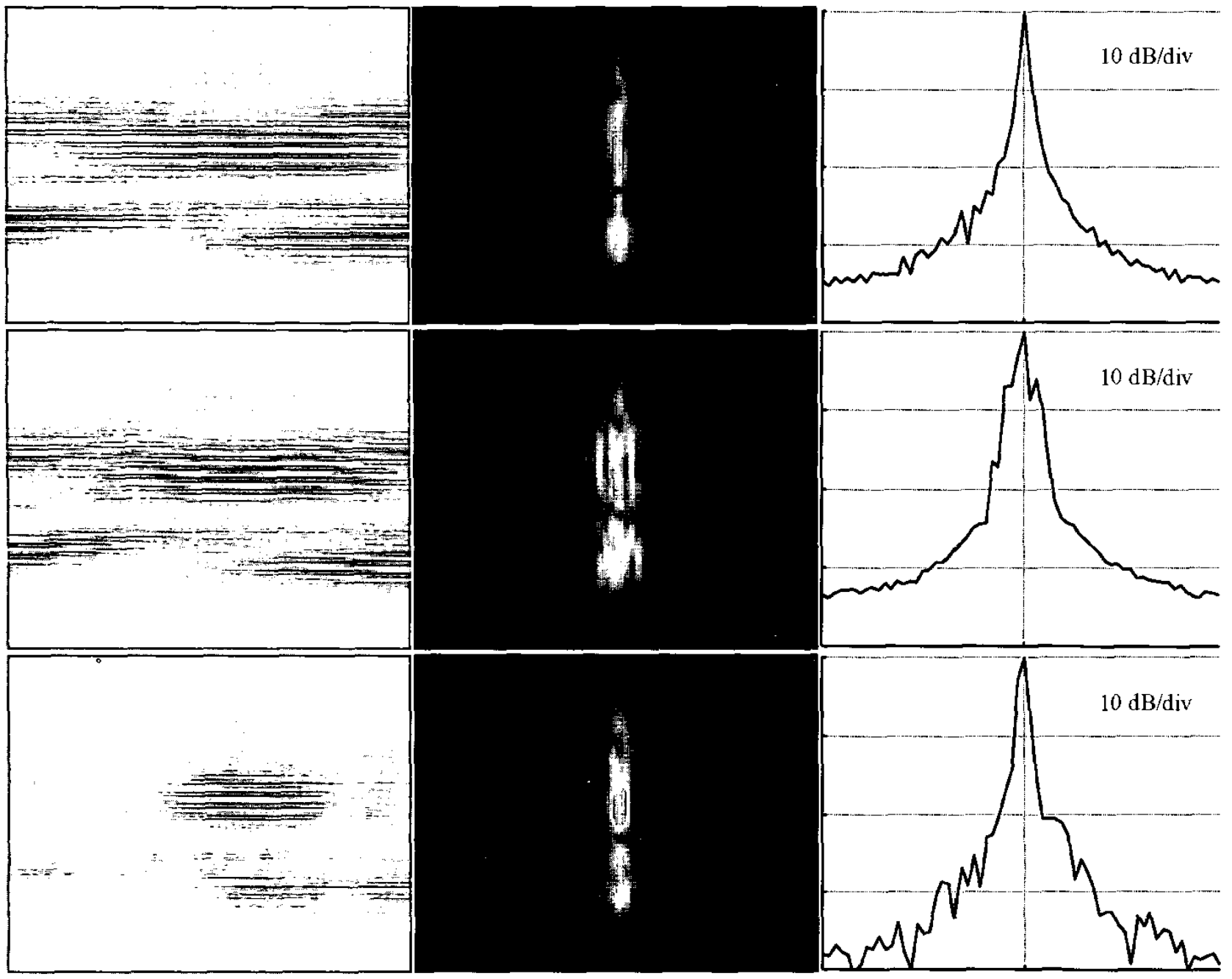

Fig. 4. Channel data and the corresponding spectrum over the aperture of diffuse scatterers. The top panels are for no phase error, and the middle and bottom pancls are for a maximum phase error of $\pi / 2$. The middle panels are for a near-field phase screen; the bottom panels are for a displaced phase screen positioned $20 \mathrm{~mm}$ from the transducer. In each case. the left panel shows the channel data, with the horizontal axis representing the channel index and the vertical axis denoting the range. The middle panel shows the spectrum at cach range. The right panel is the projection of the data shown in the middle panel.

Fig. 6(a) is the case for no aberration. Fig. 6(b) represents the case with a $\pi / 8$ maximum phasc error at the center frequency of $3.5 \mathrm{MHz}$. Fig. 6(c) shows the aberrated case with a $\pi / 4$ maximum phase error, and Fig. $6(\mathrm{~d})$ is the case with a $\pi / 2$ maximum phase error. It is obvious from Fig. 6 that the beam quality is significantly improved after adaptive weighting for each aberration case and $M_{0}$ value the far sidelobes are all significantly suppressed by more than $20 \mathrm{~dB}$. The suppression of the near sidelobe decreases as the maximum phase error and $M_{0}$ increase. Note that GCF weighting also suppresses the sidelobe level even if no aberration is present. This suggests that contrast improvement on unaberrated data also can be obtained due to reduced sidelobes. In general, the best performance of phase-aberration correction for a point target is obtained when $M_{0}$ is equal to zero (in terms of sidelobe reduction). However, the GCF-corrected beam profile with $M_{0}=0$ introduces larger variations in the near sidelobe level as indicated by the two arrows shown in Fig. 6(d). In this casc, the uncorrected beam exhibits beam splitting due to large phase aberration. The corrected beam has lower sidelobes and makes beam splitting more pronounced. Such a situation does not occur for a larger $M_{0}$. In other words, a larger $M_{0}$ (e.g., $M_{0}=1$ ) should be used to prevent such artifacts.

\section{B. Anechoic Object Simulation}

To further demonstrate the efficacy of the proposed GCF technique for targets with diffuse scatterers and potential improvement on contrast resolution, an anechoic cyst in a speckle-generating background is simulated. The center of the cyst is located at the depth of $30 \mathrm{~mm}$. The image size is $15 \mathrm{~mm}$ in the lateral direction and $10 \mathrm{~mm}$ 


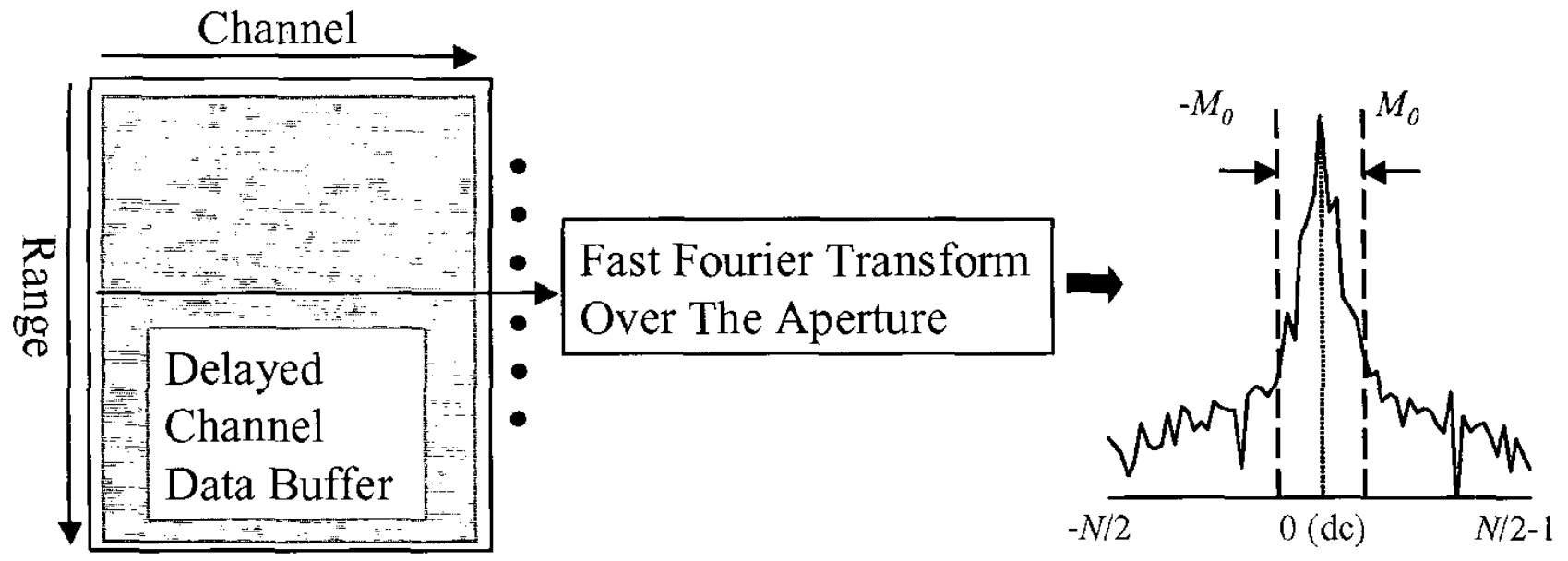

(a)

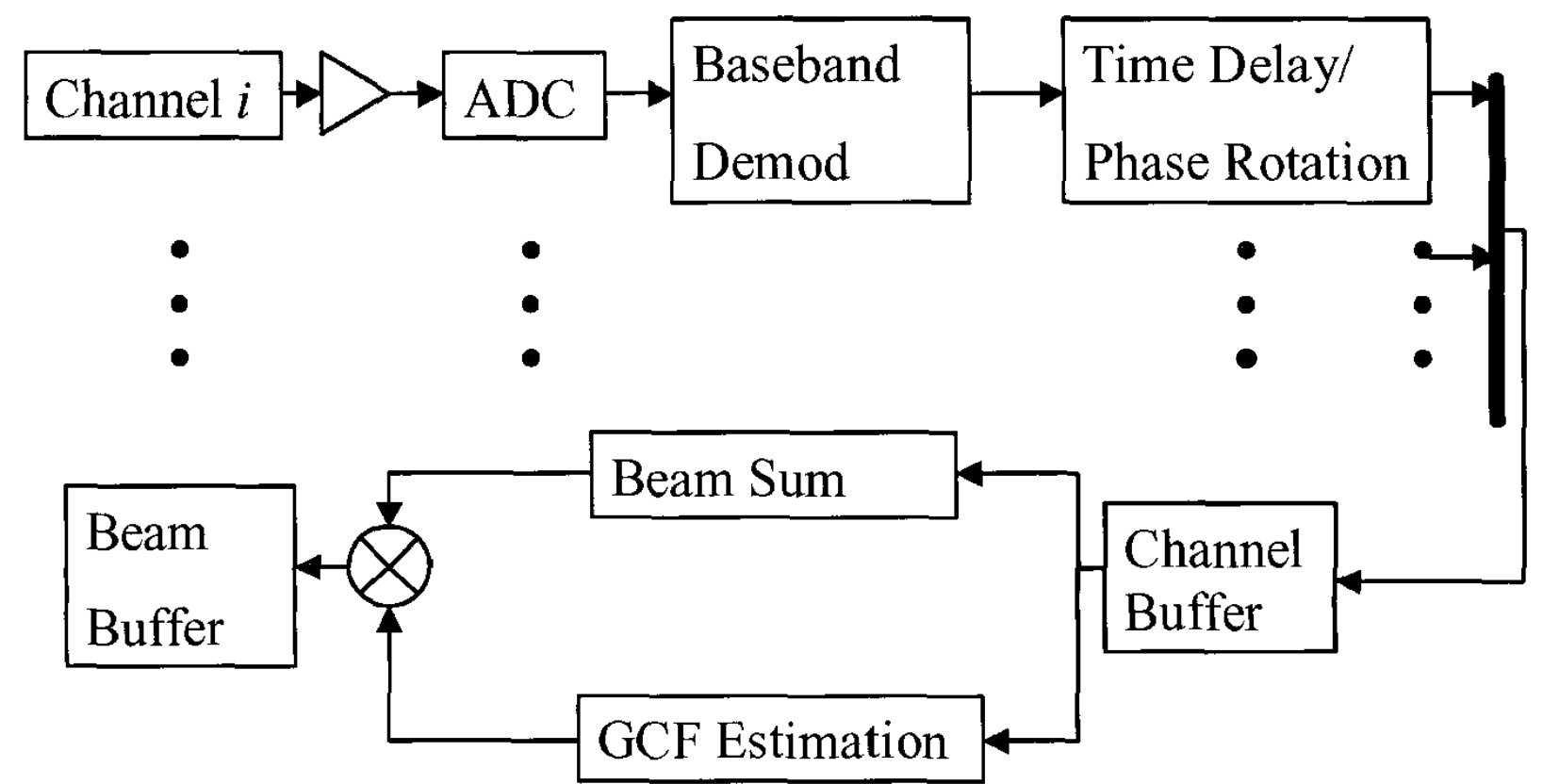

(b)

Fig. 5. (a) Schernatic showing how the GCF is calculated. (b) System block diagram for the GCF-weighting technique.

in the axial direction. The anechoic object has a diameter of $5 \mathrm{~mm}$. Positions of the scatterers are uniformly distributed, and the scattering amplitudes are Gaussian distributed. Other simulation settings are the same as those in the point target simulations for sound-velocity inhomogeneities.

Fig. 7(a) shows the simulated image under a $\pi / 4$ maximum phase error without correction. Figs. 7(b) and (c) show the corrected images with GCF weighting when $M_{0}$ was set to zero and one, respectively. Note that the images are sector-scan images prior to scan conversion and display over a $50 \mathrm{~dB}$ dynamic range. The vertical axis represents range and the horizontal axis is azimuth. Fig. 7(a) shows that the $\pi / 4$ maximum phase error results in degradation in image quality, as evidenced by the "fill in" in the anechoic cyst region. Figs. 7(b) and (c) show that detection of the cyst is improved after GCF correction for both $M_{0}$ values used in the simulations. However, the corrected image shown in Fig. 7(b) exhibits artificial black holes. The standard deviation of the image intensity was calculated in the background region indicated by the right-hand rectangular white box shown in Fig. 7(a). The standard deviation of Fig. $7(\mathrm{~b})$ is $3.94 \mathrm{~dB}$ higher than that of Fig. $7(\mathrm{a})$; the standard deviation of Fig. 7 (c) is only $1.06 \mathrm{~dB}$ higher than that of Fig. 7(a). In other words, the GCF weighting introduces large intensity variations when $M_{0}$ is set to zero. These results suggest that it is inappropriate to set $M_{0}$ to zero for diffuse scatterers. In other words, the low-frequency re- 


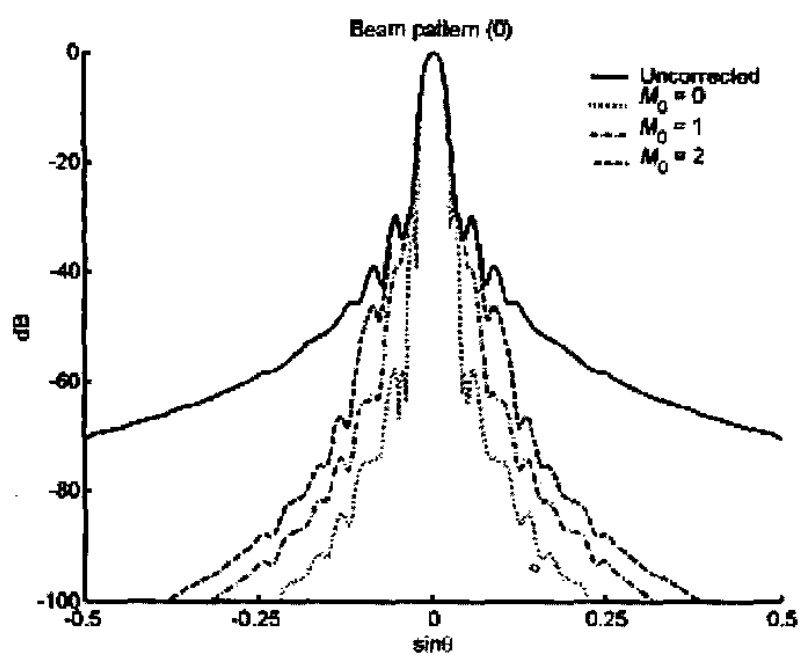

(a)

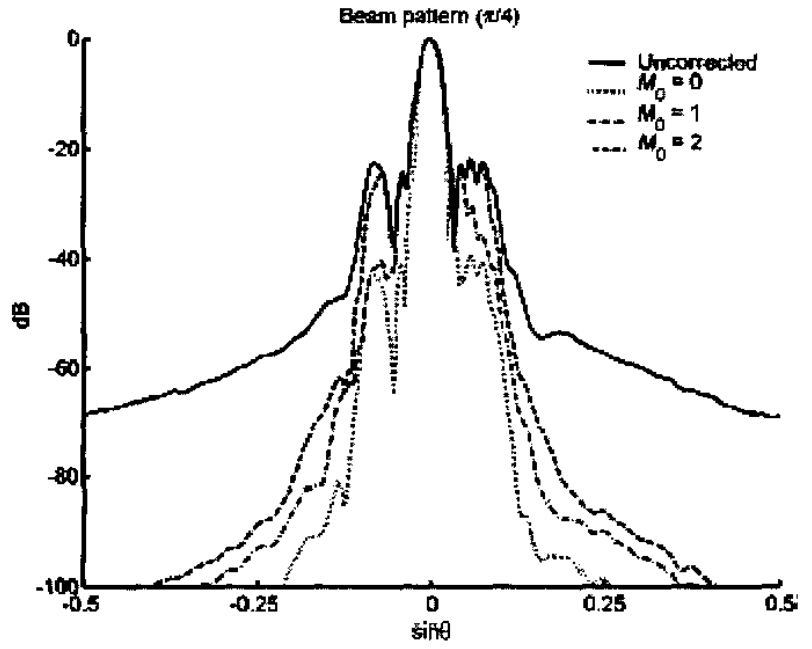

(c)

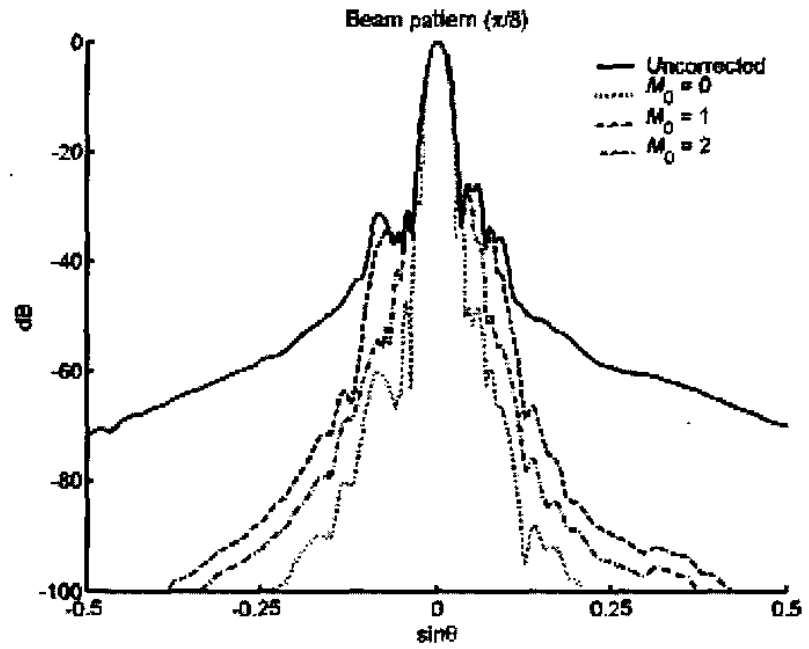

(b)

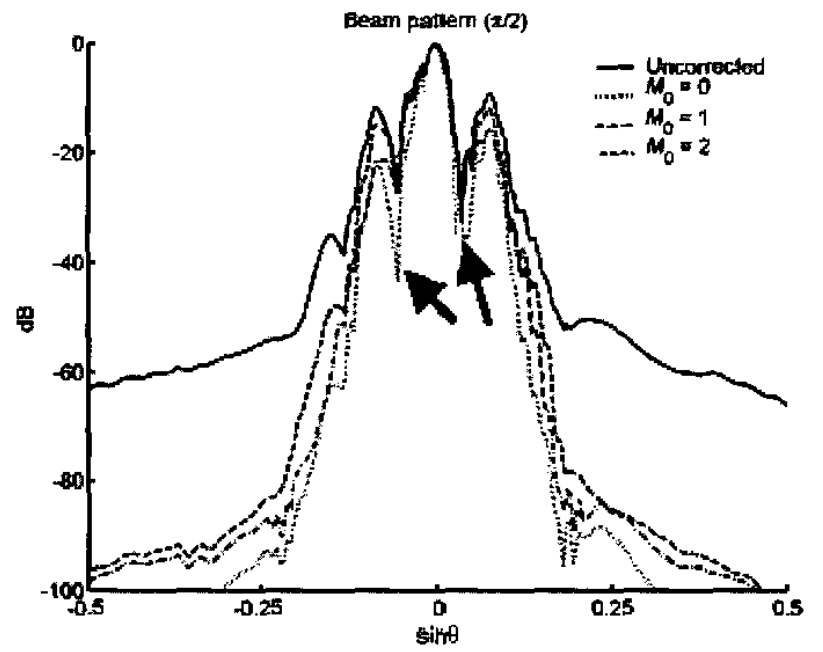

(d)

Fig. 6. Projected beam patterns for a point target. (a) No aberration. (b)-(d) $\pi / 8, \pi / 4$, and $\pi / 2$ maximum phase errors, respectively, at the center frequency of $3.5 \mathrm{MHz}$. Solid lines, uncorrectcd; dotted lines, GCF corrected with $M_{0}=0$; dashed-dotted lines, GCF corrected with $M_{0}=1$; dashed lines, GCF corrected with $M_{0}=2$.
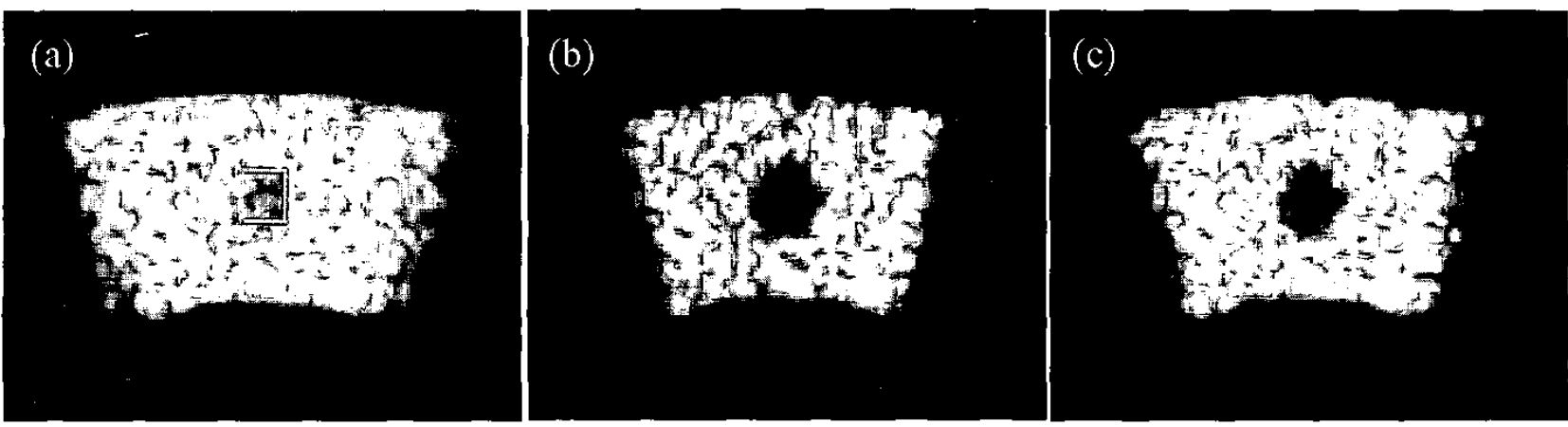

Fig. 7. Images of an anechoic cyst over a 50-dB dynamic range. The vertical axis is the range and the horizontal axis represents the azimuth. (a) $\pi / 4$ maximum phase crror at the imaging frequency of $3.5 \mathrm{MHz}$. (b) GCF-corrected inage with $M_{0}=0$. (c) GCF-corrected image with $M_{0}=1$. 


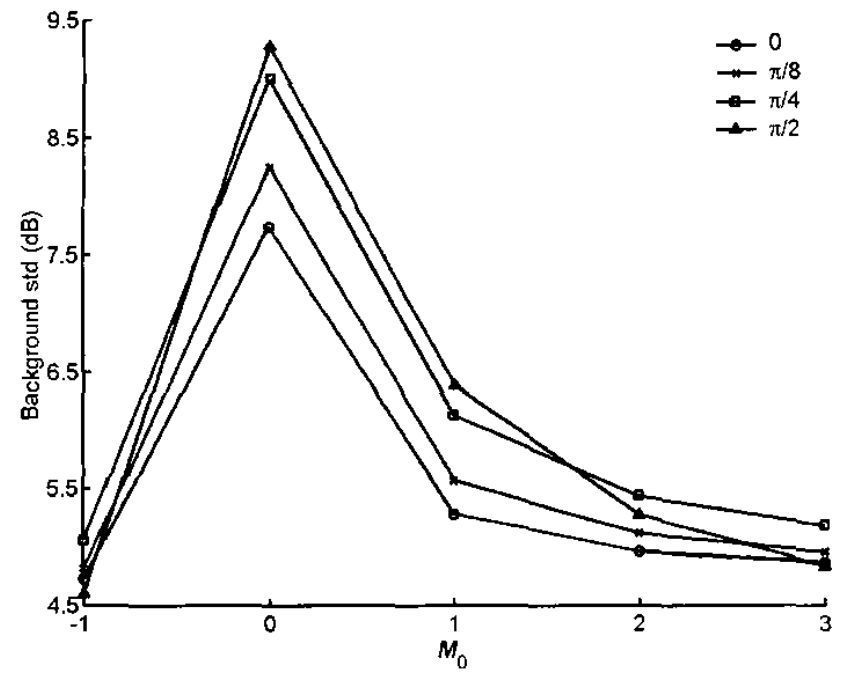

Fig. 8. Standard deviation in the background region after correction as a function of $M_{0} . M_{0}=-1$ corresponds to no correction being performed.

gion used to estimate GCF for diffuse scattcrers should be wide enough to include the inherent incoherence from a speckle-generating target. Thus, a larger $M_{0}$ is generally more preferable for this type of object.

For the four maximum phase errors-0 (i.e., no aberration), $\pi / 8, \pi / 4$, and $\pi / 2$-Fig. 8 shows the standard deviation in the background region after GCF correction as a function of $M_{0}$. Again, the standard deviation in the background is estimated from the region within the right-hand white box shown in Fig. 7 (a). Also note that a $M_{0}$ value of -1 corresponds to no GCF correction being performed. Fig. 8 indicates that the standard deviation decreases as the value of $M_{0}$ increases. In addition, for $M_{0}$ equal to zero, the corresponding standard deviation after correction is much higher than that without correction. A large standard deviation may indicate the presence of image artifacts.

Two indices related to contrast resolution are used to evaluate the improvement in image quality: the contrast ratio (CR) is defined as the ratio of the mean value in the background to the mean value in a cyst region [2], and the contrast-to-noise ratio (CNR) is defined as the CR divided by the standard deviation of image intensity in the background region [3] (note that the background and cyst regions are indicated by the right- and left-hand white boxes shown in Fig. 7(a), respectively). Fig. 9 shows the CR after correction as a function of $M_{0}$ for different maximum phase crrors. Fig. 9 shows that the $\mathrm{CR}$ decreases as $M_{0}$ increases. Moreover, the contrast enhancement decreases as the maximum phase error increases. The CNR after correction as a function of $M_{0}$ for the same four maximum phase errors is shown in Fig. 10, which indicates that the best improvement of CNR using GCF generally can be achieved when $M_{0}$ is equal to one. Again, such a result suggests the inherent incoherence from a speckle-generating target should be taken into consideration to choose a proper $M_{0}$. For a

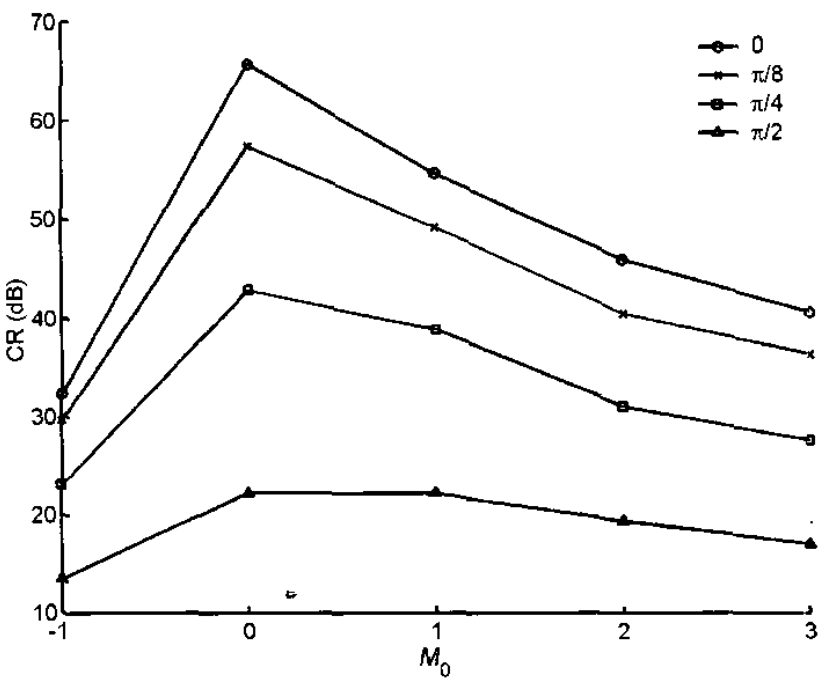

Fig. 9. CR after correction as a function of $M_{0}$ for different maximum phase errors.

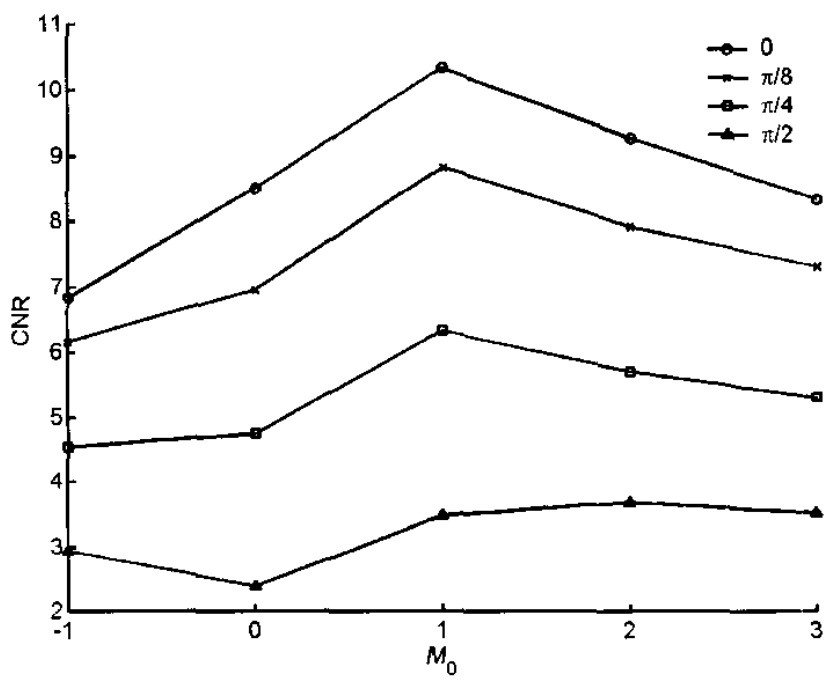

Fig. 10. CNR after correction as a function of $M_{0}$ for different maximum phase errors.

given value of $M_{0}$, the improvement of CंNR decreases as the maximum phase error increases. Furthermore, for $M_{0}$ equal to zero, the corresponding CNR after correction generally increases except for the case with a $\pi / 2$ maximum phase error. This may be caused by the beam splitting effect with $M_{0}=0$ in Fig. 6(d). The results shown in Figs. 9 and 10 indicate that both CR and CNR may need to be considered when evaluating the contrast improvement.

Figs. 11(a)-(c) and (d)-(f) show the uncorrected and GCF-corrected images with maximum phase errors of 0 . $\pi / 4$, and $\pi / 2$, respectively. Based on Fig. $10, M_{0}=1$ was used for GCF correction. It is obvious that the detection of the cyst is significantly improved, but the adaptive weighting technique is less effective for the case with a $\pi / 2$ maximum phase error. The CR and CNR values for the 

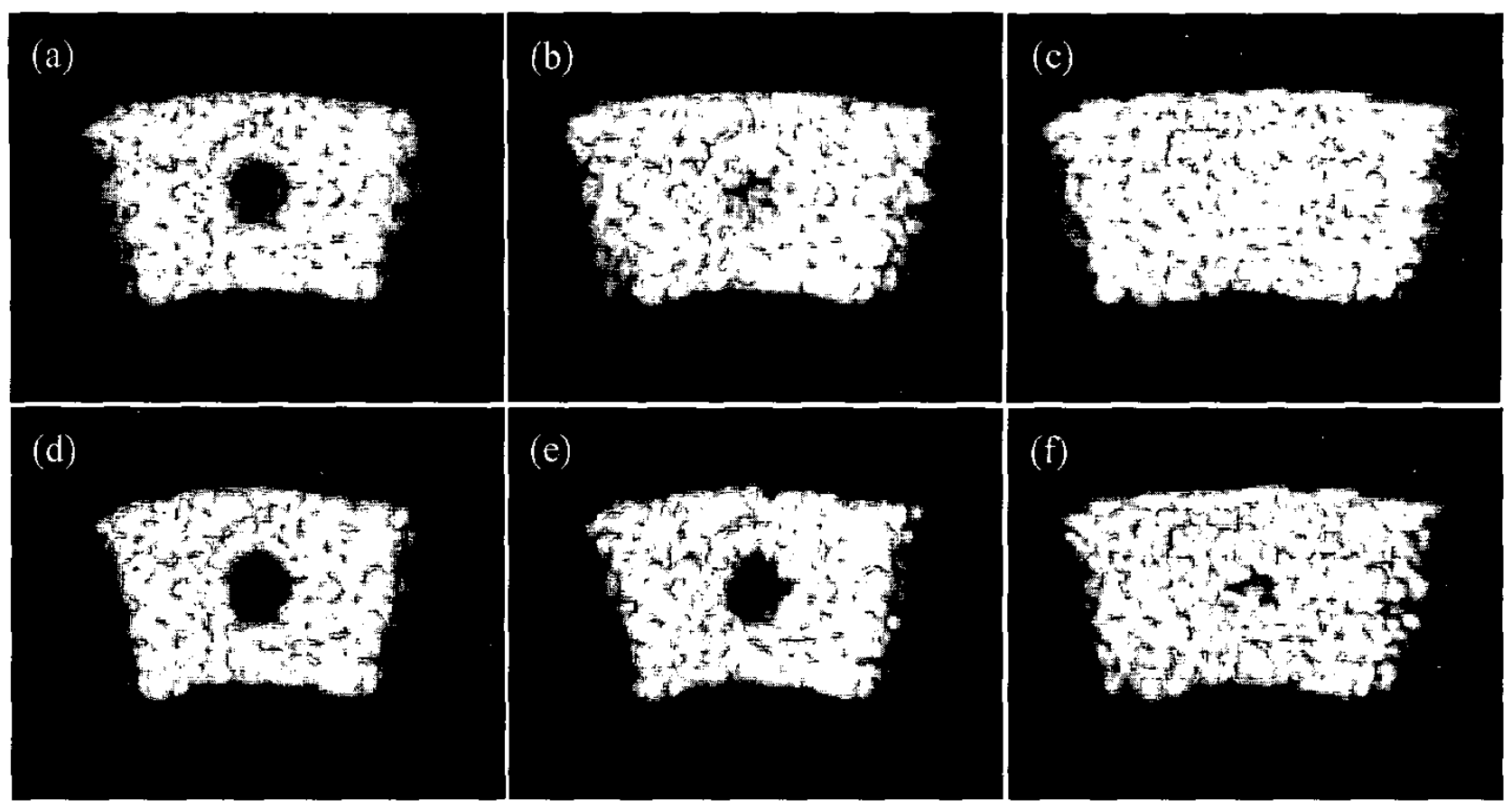

Fig. 11. Original and GCF-corrected images of a phantom with an anechoic cyst over a 50-dB dynamic range. The top row shows the uncorrected images; the bottom row shows the corrected images. (a) and (d) No aberration. (b) and (e) $\pi / 4$ maximum phase error at the imaging frequency of $3.5 \mathrm{MHz}$. (c) and (f) $\pi / 2$ maximum phase crror.

TABLE I

CR and CNR for the Simulated Images of a Phan'tom with AN AneCholc Cyst.*

\begin{tabular}{cccc}
\hline \multicolumn{4}{c}{$\mathrm{CR}(\mathrm{dB})$} \\
\hline & 0 & $\pi / 4$ & $\pi / 2$ \\
\hline $\begin{array}{c}\text { Uncorrected } \\
\text { GCF }\end{array}$ & 32.32 & 23.07 & 13.54 \\
& 54.67 & 38.81 & 22.18 \\
\hline & $\mathrm{CNR}$ & & \\
\hline & 0 & $\pi / 4$ & $\pi / 2$ \\
\hline Uncorrected & 6.84 & 4.55 & 2.94 \\
GCF & 10.34 & 6.33 & 3.47 \\
\hline
\end{tabular}

*0 means no aberration. $\pi / 4$ and $\pi / 2$ represent maximum phase errors, respectively, at the imaging frequency of $3.5 \mathrm{MHz}$.

images in Fig. 11 are listed in Table I. Using GCF weighting, the corrected images exhibit contrast improvements of $22.35 \mathrm{~dB}, 15.74 \mathrm{~dB}$, and $8.64 \mathrm{~dB}$ for maximum phase errors of $0, \pi / 4$, and $\pi / 2$, respectively. A higher. CNR also is obtained after adaptive GCF weighting. Note that the CR and CNR of the unaberrated case also increase after the GCF weighting. This is because the sidelobe lcvel of an unaberrated beam also is suppressed by the GCF weighting, as shown in Fig. 6(a).

\section{Experimental Results}

Three complete RF data sets with three different levels of distortion were used to test the efficacy of the pro- posed adaptive weighting mothod (all the raw data are available from http://bul.eecs.umich.edu). The data sets were the same as those used by O'Donnell and Flax [2]. The first data set was RF data acquired from a tissuemimicking phantom without distortion. The other two data sets were acquired from the same phantom, except that $1 \times$ and $2 \times$ distortion were introduced, corresponding to maximum phase errors of $\pi$ and $2 \pi$, respectively, at the imaging frequency of $3.33 \mathrm{MHz}$. The distortion resulted from a room-temperature vulcanizing silicon rubber plate inserted between the array and the tissuc-mimicking phantom. They were acquired using a 64-element, 3.33$\mathrm{MHz}$ phased-array transducer with a $17.76-\mathrm{MHz}$ sampling frequency. Dynamic receive focusing with an $\mathrm{f} /$ number of 2 was applied, and the transmit focus was at $60 \mathrm{~mm}$. All images arc displayed over a $50 \mathrm{~dB}$ dynamic range and are shown in a prescan conversion format (i.e., the vertical axis represents range and the horizontal axis is azimuth).

Fig. 12(a) is the image without distortion, and Figs. 12(b) and (c) show images of the phantom with $1 \times$ and $2 \times$ distortion, respectively. Figs. 12(d)-(f) show the corrected images of Figs. 12(a)-(c), respectively, using GCF weighting with $M_{0}$ set to 2 . Note that all the images are normalized by the mean value of the homogeneous region located at the image center. These corrected images show that the image quality is noticeably improved. Table II lists the CR and CNR values for the images shown in Fig. 12. The two parameters are calculated using the background and cyst region indicated by the black and white boxes shown in Fig. 12(a). Using GCF, the corrected 


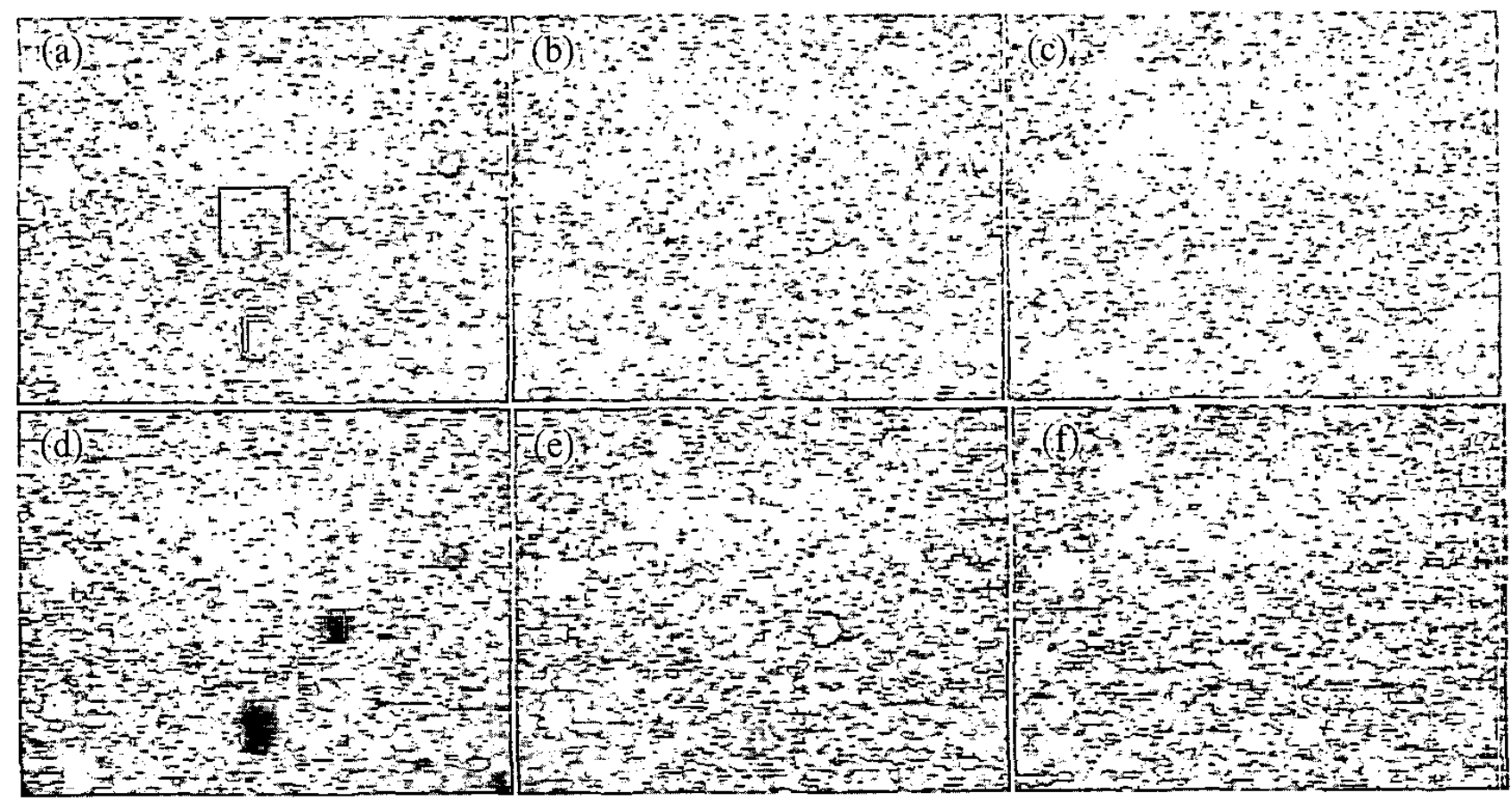

Fig. 12. Original and GCF-corrected images of a tissue-mimicking phantom over a 50-dB dynamic range. The top row shows the uncorrected images; the bottom row shows the corrected images. (a) and (d) No distortion. (b) and (e) $1 \times$ distortion. (c) and (f) $2 \times$ distortion.

TABLE II

CR and CNR for the Images of a Tissue-Mimicking Phantom.

\begin{tabular}{|c|c|c|c|}
\hline \multicolumn{4}{|c|}{$\mathrm{CR}(\mathrm{dB})$} \\
\hline & No distortion & $1 \times$ distortion & $2 \times$ distortion \\
\hline Uncorrected & 25.44 & 16.86 & 11.04 \\
\hline GCF & 40.67 & 27.97 & 18.09 \\
\hline \multicolumn{4}{|c|}{$\mathrm{CNR}$} \\
\hline & No distortion & $1 \times$ distortion & $2 \times$ distortion \\
\hline Uncorrected & 4.54 & 2.95 & 1.97 \\
\hline GCF & 6.41 & 3.65 & 2.30 \\
\hline
\end{tabular}

images have contrast enhancements of $15.23 \mathrm{~dB}$ (no distortion), $11.11 \mathrm{~dB}(1 \times)$, and $7.05 \mathrm{~dB}(2 \times)$. Higher CNR values also were obtained after adaptive weighting, and the corrected images with less distortion have both higher CR and higher CNiR values.

\section{Discussion}

The simulation results show that the cutoff frequency $M_{0}$ affects the performance of the GCF technique. Weighting by the coherence factor (i.e., GCF with $M_{0}=0$ ) is only suitable for point targets, but it may make the beamsplitting effect more pronounced. Hence, a larger $M_{0}$ (i.e. $M_{0} \neq 0$ ) should be used. In addition, for diffuse scatterers, $M_{0}$ also should be sufficiently large in order not to introduce image artifacts. However, the GCF technique becomes less effective when $M_{0}$ is too large. Based on the results shown in this paper, the proper value of $M_{0}$ for diffuse scatterers is $1-3$.
Characteristics of the GCF are further explored by numerical simulations and compared to the original $\mathrm{CF}$. It was previously demonstrated that the $\mathrm{CF}$ approaches to $2 / 3$ for one-dimensional arrays when the number of channels is sufficiently large and when there is no focusing error [14]-[16]. For GCF, the coherent sum depends on the specific $M_{0}$ used in the calculations, and an analytical solution is not likely to exist. Therefore, numerical simulations are used to study the behavior with different cutoff frequencies. For each case, simulations were performed based on 10 different realizations of the diffuse-scatterer distributions, and mean values and standard deviations were calculated (shown in Figs. 13(a) and (b), respectively). In Fig. 13 the solid lines correspond to $M_{0}=0$ (i.e., the original $\mathrm{CF}$ ), the dotted lines are for $M_{0}=1$, and the dashed-dotted lines are for $M_{0}=2$. Fig. 13 shows that: as $M_{0}$ increases, the mean increases irrespective of the number of channels. However, the standard deviation decreases as $M_{0}$ increases. In other words, the CF (i.e., GCF with $M_{0}=0$ ) has a larger variation. Such a variation may cause image artifacts after the CF weighting. This agrees with the previous results.

The GCF is defined here without taking noise into consideration. In real imaging situations the signal-to-noise ratio (SNR) decreases as the imaging depth increases due to tissue attenuation, so the estimated GCF may be affected by noise. Assume that $S_{\text {low }}$ and $N_{\text {low }}$ are the spectral energies of the signals and noise within the prespecified low-frequency range, respectively, and that $S_{\text {total }}$ and $N_{\text {total }}$ are the total spectral energies of the signals and noise. The relationship between GCF without noise (i.e., 


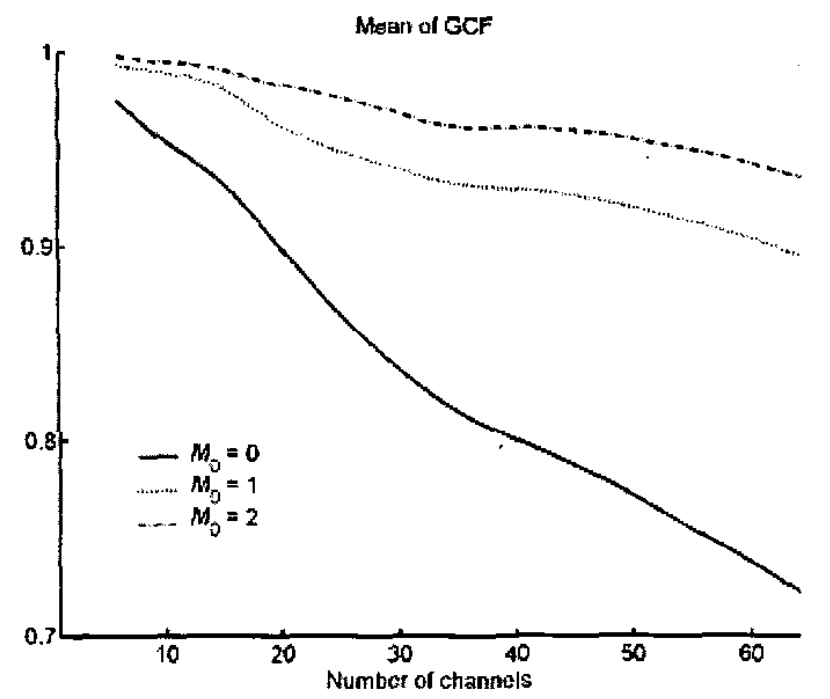

(a)

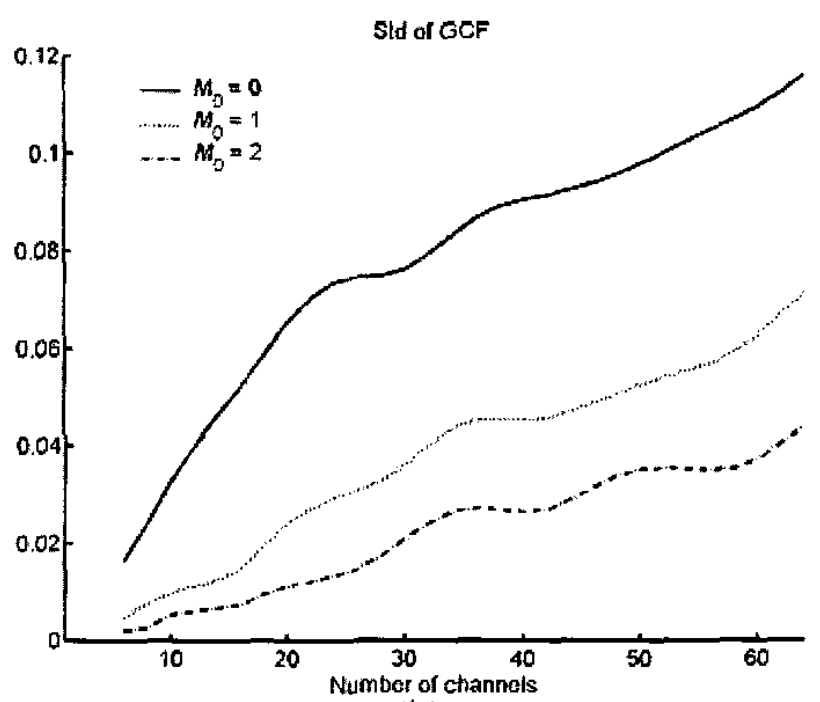

(b)

Fig. 13. Simulated GCF with 10 different realizations of scatterer distributions. The solid line is for $M_{0}=0$, the dotted line is for $M_{0}=1$, and the dashed-dotted line is for $M_{0}=2$. (a) and (b) show the mean and standard deviation values, respectively.

an infinite SNR) and the estimated GCF with a finite SNR (denoted by $\mathrm{GCF}^{\prime}$ ) can be obtained as follows:

$$
\begin{aligned}
\mathrm{GCF}^{\prime} & =\frac{S_{\text {low }}+N_{\text {low }}}{S_{\text {total }}+N_{\text {total }}} \\
& =\frac{N_{\text {total }}\left(\frac{S_{\text {low }}}{N_{\text {total }}} \cdot \frac{S_{\text {total }}}{S_{\text {total }}}+\frac{N_{\text {low }}}{N_{\text {total }}}\right)}{N_{\text {total }}(1+\mathrm{SNR})} \\
& =\frac{\left(\frac{S_{\text {low }}}{S_{\text {total }}} \cdot \mathrm{SNR}+\frac{N_{\text {low }}}{N_{\text {total }}}\right)}{1+\mathrm{SNR}} \\
& =\frac{\left(\mathrm{GCF} \cdot \mathrm{SNR}+\frac{N_{\text {low }}}{N_{\text {total }}}\right)}{1+\mathrm{SNR}} \\
& =\frac{\left(\mathrm{GCF} \cdot \mathrm{SNR}+\frac{2 M_{0}+1}{N}\right)}{1+\mathrm{SNR}},
\end{aligned}
$$

where $\mathrm{SNR}=\frac{S_{\text {total }}}{N_{\text {total }}}$, and $N$ is the number of points used for the FFT. If the SNR is very low, the estimated GCF can be expressed as:

$$
\mathrm{GCF}^{\prime} \approx \frac{\left(2 \cdot M_{0}+1\right)}{N}
$$

For the experimental data used in this paper, $M_{0}$ was set to 2 and $N$ was 64 . From (7), the estimated GCF should be about 0.078 when the SNR is very low. This value is close to the experimental value of 0.082 . However, the penetration depth can be defined as the depth in which the SNR is equal to 1 . In this case, the corresponding estimated GCF can be expressed as:

$$
\begin{aligned}
\mathrm{GCF}^{\prime} & =\frac{\left(\mathrm{GCF}+\frac{2 M_{0}+1}{N}\right)}{2} \\
& =\frac{\mathrm{GCF}+0.078}{2} \\
& \approx \frac{\mathrm{GCF}}{2}\left(\mathrm{e} . \mathrm{g} ., N=64, M_{0}=2\right) .
\end{aligned}
$$

The (8) shows that the estimated GCF at the penetration depth (defined by SNR =1) is half the ideal GCF. The above results also show that, although the estimated GCF is dependent on the SNR, the real GCF that needs to be used for adaptive weighting still can be retrieved if the SNR can be estimated. In addition, $\mathrm{GCF}^{\prime}$ also can be applied directly in order to reduce the effects of a low SNR.

Performance of the proposed GCF tcchnique with a displaced phase screen is evaluated compared with the correlation-bascd technique [1]: [2] and PARCA2 [10]. The simulation method is the same as that used by $\mathrm{Ng}$ et al. [5]. The displaced phase-screen profile is the same as the normalized phase-aberration profile shown in Fig. 2. A sector scan from $-5^{\circ}$ to $5^{\circ}$ was performed. The maximum phase error is $\pi / 2$ at the center frequency of $3.5 \mathrm{MHz}$. A point source is located at a depth of $80 \mathrm{~mm}$, with the transducer being focused at the same depth; the simulation results are shown in Fig. 14. Figs. 14(a) and (c) show the simulation results of the normalized receive beam patterns in the presence of a phase screen at $0 \mathrm{~mm}$ from the transduccr, and Figs. 14(b) and (d) show the receive bcam patterns with the same phase screen at $40 \mathrm{~mm}$. The lateral axis is azimuth. Because a point target is imaged, $M_{0}$ is chosen as 0 for GCF calculations.

Figs. 14(a) and (b) compare the GCF and correlationbased techniques. As expected, the latter tcchnique performs very well for the near-field phase screen [Fig. 14(a)]. The GCF-corrected beam pattern is improved, exhibiting lower sidelobes and a narrower mainlobe. However, the GCF-corrected beam introduces large variations in sidelobe level. This can be avoided by using a larger $M_{0}$ (e.g., $M_{0}=1$ ) with less sidelobe reduction. Fig. 14(b) shows that the GCF technique performs well for a displaced phase screen, outperforming the correlation-based technique for the displaced phase screen situation. Figs. 14(c) and (d) 


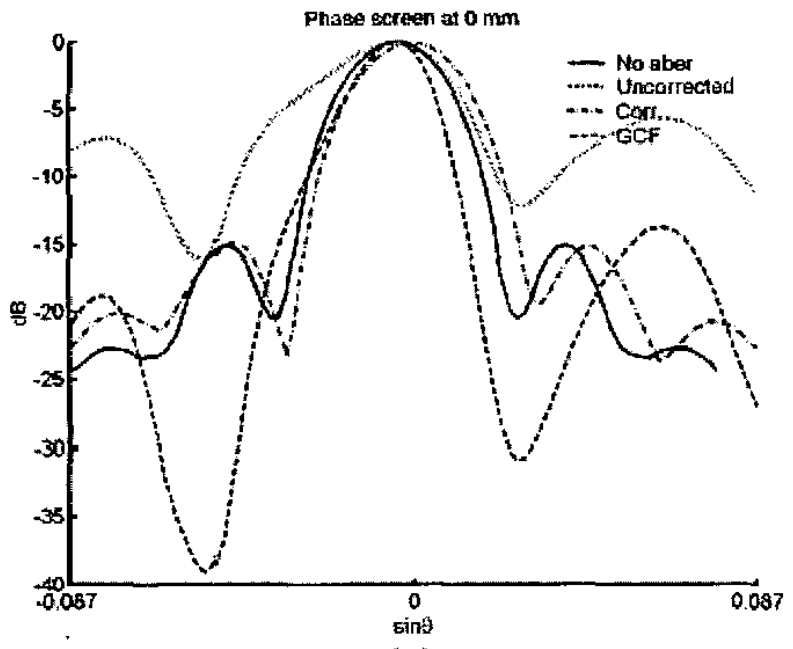

(a)

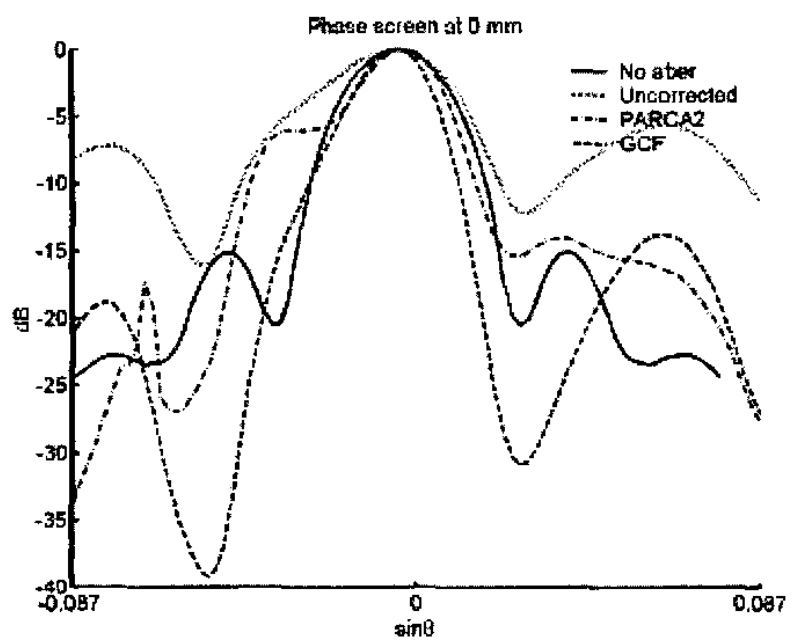

(c)

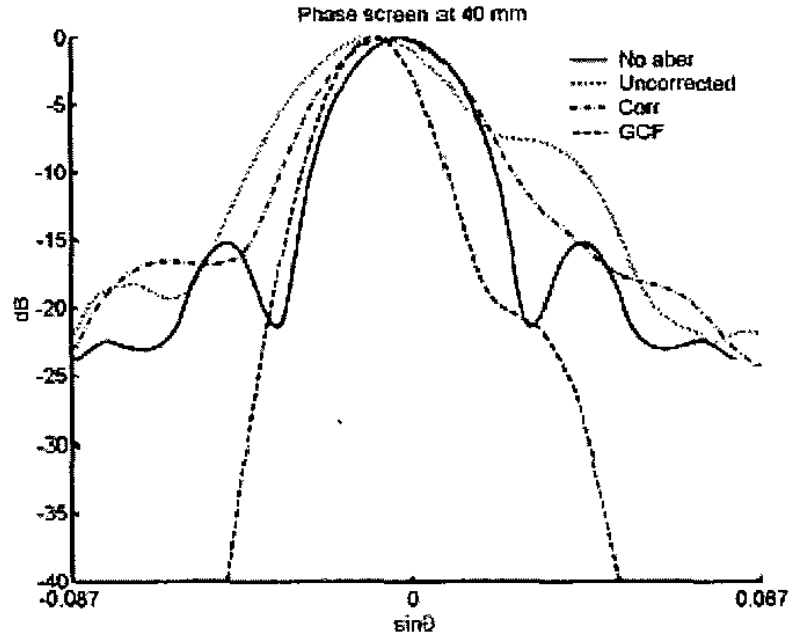

(b)

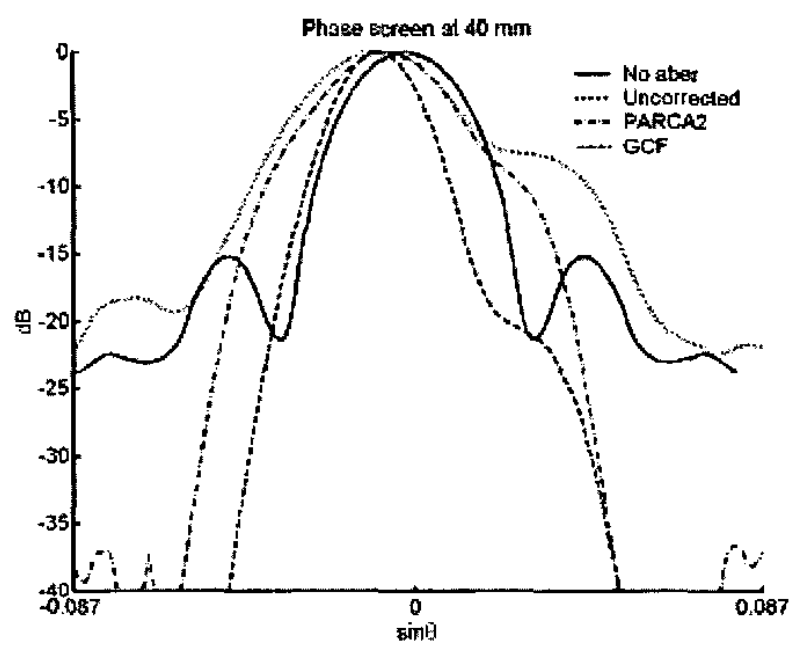

(d)

Fig. 14. Projected receive bean patterns for a point target with a displaced phase screen. (a) and (c) Phase screen at $0 \mathrm{~mm}$. (b) and (d) Phase screen at $40 \mathrm{~mm}$. Solid lines, no aberration; dotted lines, uncorrected; dashed lines, corrected by GCF weighting; dashed-dotted lines, corrected by the correlation-based technique in (a) and (b), and corrected by PARCA2 in (c) and (d).

compare the GCF technique and PARCA2 (three iterations were used for PARCA2). Fig. 14(c) shows that, for the near-ficld phase-screen situation, the PARCA2corrected beam pattern has higher sidelobes than the GCF-weighted beam pattern but with less variations. Fig. 14(d) shows that the GCF technique also outperforms PARCA2 for the displaced phase-screen situation.

The correlation-based technique using one-dimensional arrays has shown limited success due to inadequate spatial sampling of sound-velocity inhomogeneities in elevation. Two-dimensional arrays have been suggested for obtaining adequate performance in clinical situations [6]. Unlike the correlation-based technique, however, the proposed GCF technique only attempts to improve beam quality regardless of the dimensionality of the array. Therefore, for real-time applications using one-dimensional arrays; the proposed GCF technique is more suitable than the correlation-based technique. Compared to PARCA2, the GCF technique has lower computational complexity and memory requirements, and no iterations are needed.

\section{Conclusions}

This paper proposes an adaptive weighting technique based on the GCF for reducing focusing errors in acoustic imaging, especially for the focusing error caused by sound-velocity inhomogeneities. The technique is independent of the source of the artifacts and can be implemented efficiently. Thus, it also can be applied to reduce the focusing-delay accuracy, extend the transmit focal zone, or minimize the effects of sound-velocity inhomogeneities. Especially for sound-velocity inhomogencities, 
the simulation and experimental results demonstrate that the proposed GCF technique corrects for both near-field and displaced-phase errors. Furthermore, it is shown that the SNR and the cutoff frequency $M_{0}$ influence the performance of the proposed GCF technique. The proposed GCF technique performs better than PARCA2, and its computational complexity and memory requirements are lower. In addition, no iteration is needed for the GCF technique. The proposed technique also performs better than the correlation-based techmique when a displaced phase screen is present. The proposed GCF technique can be incorporated into current medical ultrasonic imaging systems with only modest modifications.

\section{ACKNOWLEDGMEN'TS}

Data used in this paper were downloaded from the Biomedical Ultrasonics Laboratory at the University of Michigan (http://bul.eecs.umich.edu). The authors also would like to thank C.-C. Shen and Dr. K. Ustuner for general discussions. The authors also acknowledge the comments made by the reviewers.

\section{REFERENCES}

[1] S. W. Flax and M. O'Donncll, "Phase-aberration correction using signals from point reflectors and diffuse scatterers: Basic principles," IEEE Trans. Ultrason., Ferroelect., Freq. Contr., vol. 35, no. 6 , pp. $758-767,1988$.

[2] M. O'Donnell and S. W. Flax, "Phase-aberration correction using signals from point reflectors and diffuse scattercrs: Measurements," IEEE Trans. Ultrason., Ferroclect., Freq. Contr., vol. 35 , no. 6, pp. 768-774, 1988.

[3] S. Krishnan, K. W. Rigby, and M. O'Donnell, "Improved estimation of phase aberration profile," IEEE Trans. Ultrason. Ferroelect., Freq. Contr., vol. 44, no. 3, pp. 701-713, 1997.

[4] D. L. Liu and R. C. Waag, "Correction of ultrasonic wavefront distortion using backpropagation and a reference waveform method for time-shift compensation," J. Acoust. Soc. Amer., vol. 96, no. 2, pp. 649-660, 1994 .

[5] G. C. Ng, P. D. Freiburger, W. F. Walker, and G. E. Trahey, "A speckle target adaptive imaging technique in the presence of distributed aberrations," IEEE Trans. Ultrason., Ferroelect., Freq. Contr., vol. 44, no. 1, pp. 140-151, 1997.

[6] P.C. Li and M. O'Donnell, "Phase aberration correction on twodimensional conformal arrays," IEEE Trans. Ultrason., Ferroelect., Freq. Contr., vol, 42, no. 1, pp. 73-82, 1995.

[7] P.C. Li, S. W. Flax, E. S. Ebbini, and M. O'Domnell, "Blocked element compensation in phased array imaging;" IEEE Trans. Ultrason., Ferroelect., Freq. Contr., vol. 40, no. 4, pp. 283-292, 1993.

[8] P.-C. Li and M. O'Donnell, "Improved detectability with blocked element compensation," Ultrason. Imag., vol. 16, no. 1, pp. 1-18, 1994.

[9] S. Krishnan, P.-C. Li, and M. O'Donnell, "Adaptive compensation of phase and magnitude aberrations." IEEE Trans. Ultrason., Ferroelest., Freq. Contr., vol. 43, no. 1, pp. 44-55, 1996.

[10] S. Krishnan, K. W. Rigby, and M. O'Donnell, "Efficient parallel adaptive aberration correction," IEEE Trans. Ultrason., Ferroelect., Freq. Contr., vol. 43, no. 1, pp. 691-703, 1998.

[11] D.-L. D. Liu, P. Von Behren, and J. Kim, "Single transmit imaging." in Proc. IEEE Ultrason. Symp., 1999, pp. 1275-1278.
[12] M. O'Donnell, "Efficient parallel receive beam forming for phased array imaging using phase rotation," in Proc. IEEE Ultrason. Symp., 1990, pp. 1495-1498.

[13] M. K. Jeong, "A Fourier transform-based sidelobe reduction method in ultrasound imaging," IEEE Trans. Ultrason., Ferroelect., Freq. Contr., vol. 47, no. 3, pp. 759-763, 2000.

[14] K. W. Hollman, K. W. Rigby, and M. O'Donnell, "Coherence factor of speckle from a multi-row probe," in Proc. IEEE Ultrason. Symp., 1990, pp. 1257-1260.

[15] R. Mallart and M. Fink, "Adaptive focusing in scattering media through sound-speed inhomogeneities: The van Cittert-Zernike approach and focusing criterion," J. Acoust. Soc. Amer, vol. 96, no. 6, pp. 3721-3732, 1994.

[16] S. D. Silverstein, "Ultrasound scattering model: 2-D crosscorrelation and focusing criteria-theory, simulations, and experiments," IEEE Trans. Ultrason., Ferroelect., Freq. Contr., vol. 48, no. 4, pp. 1023-1030, 2001.

[17] A. V. Oppenheim, R. W. Schafer, and J. R. Buck, Discrete-Time Signal Processing, 2nd ed. Englewood Cliffs, NJ: Prentice-Hail, 1998.

[18] J. A. Jensen, "Field: A program for simulating ultrasound systerns," Med. Biol. Eng. Comput, vol. 34, pp. 351-359, 1996.

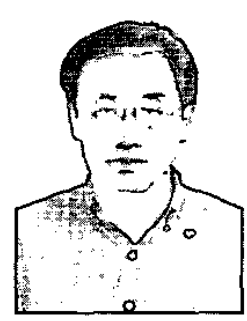

Pai-Chi Li (S'93-M'95-SM'01) received the B.S. degree in electrical engineering from National Taiwan University, Taipei, Taiwan, R.O.C. in 1987, and the M.S. and Ph.D. degrees from the University of Michigan, Ann Arbor in 1990 and 1994, respectively, both in electrical engineering: systems.

He was a research assistant with the Department of Electrical Engineering and Computer Science from 1990 to 1994 . He joined Acuson Corporation, Mountain View, CA, as a member of the Technical Staff in June 1994. His work in Acuson was primarily in the areas of medical uitrasonic imaging system design for both cardiology and general imaging applications. In August 1997, he went back to the Department of Electrical Engineering at National Taiwan University as Assistant Professor. He then became Associate Professor in August 1998. His current research interests include biomedical ultrasonic imaging and signal processing.

Dr. $\mathrm{Li}$ is a senior member of IEEE and Associate Editor of IEEE Transactions on Ultrasonics, Ferroelectrics, and Frequency Control. He received the 2002 Dr. Wu Dayou Rescarch Award from National Science Council, the 2002 Outstanding Young Electrical Engineer Award from Chinese Institute of Electrical Engineering and the 2001 Distinguished Industrial Collaboration Award of Ministry of Education. He was also the recipient of the Distinguished Achievement Award in Electrical Engineering: Systems in 1994 for his outstanding academic achievement at the University of Michigan.

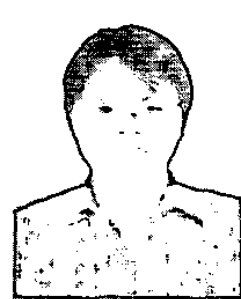

Meng-Lin Li (S'02) was born in 1976 in Tainan, Taiwan, R.O.C. He received the B.S. degree in electrical engineering from National Taiwan University, Taipei, Taiwan, R.O.C. in 1999 . He is currently working toward the $\mathrm{Ph} . \mathrm{D}$. degree in electrical engineering at $\mathrm{Na}-$ tional Taiwan University. His current research interests include biomedical ultrasonic imaging, especially for synthetic aperture focusing and adaptive imaging, and signal processing. $\mathrm{He}$ is a student member of IEEE. 